\title{
Human Urine-Derived Stem Cells Improve Partial Bladder Outlet Obstruction in Rats: Preliminary Data And microRNA-mRNA Expression Profile
}

\section{Menjiang Tu ( $\sim$ jefferstoto@hotmail.com)}

Shenzhen University General Hospital https://orcid.org/0000-0002-1260-0033

\section{Rui Wang}

Shenzhen University General Hospital

Pei Zhu

Shenzhen University General Hospital

\section{Qingqing Wang}

Third Military Medical University Second Affiliated Hospital: Xinqiao Hospital

\section{Bishao Sun}

Third Military Medical University Second Affiliated Hospital: Xinqiao Hospital

\section{Keshi Lu}

Shenzhen University General Hospital

Jiawei Zhang

Shenzhen University General Hospital

\section{Weijie Xie}

Shenzhen University General Hospital

\section{Huan Guo}

Shenzhen University General Hospital

\section{Shulin Li}

Shenzhen University General Hospital

\section{Yuqi Wu}

Shenzhen University General Hospital

\section{Xiangwei Wang}

Shenzhen University General Hospital

\section{Research Article}

Keywords: partial bladder outlet obstruction, human urine-derived stem cells, gene expression profiles, microRNA, miR-142, miR-9a

Posted Date: November 1st, 2021 
DOl: https://doi.org/10.21203/rs.3.rs-838807/v2

License: (c) (1) This work is licensed under a Creative Commons Attribution 4.0 International License. Read Full License

Version of Record: A version of this preprint was published at Stem Cell Reviews and Reports on March 1st, 2022. See the published version at https://doi.org/10.1007/s12015-022-10340-0. 


\section{Abstract}

Partial bladder outlet obstruction (pBOO) often results in bladder tissue inflammation and remodeling. As human urine-derived stem cells (USCs) have demonstrated therapeutic benefits, we used a rat model to investigate the effect of USCs on bladder function and explore the miRNA and gene expression profiles in bladder tissue using RNA sequencing. Eighteen rats were assigned to a sham surgery group, pBOO group, and pBOO+USC group (six biweekly treatments). Routine urodynamic monitoring, analysis of detrusor muscle strips, and pathophysiology assessments were conducted. Finally, altered miRNA and mRNA expression profiles of bladder tissue were examined using RNA sequencing and bioinformatics analysis. After USC treatment, elevated bladder compliance and maximal voiding pressure, declined end filling pressure and voided volume, and improved detrusor muscle contractility and carbachol sensitivity were found. Histology and TUNEL assay revealed reduced collagen deposition and muscle cell apoptosis in bladder tissue. The differential expression of eight miRNAs was reversed by USC treatment. Two large nodes (miR-142 and miR-9a) were identified in the miRNA-gene interaction network in the USC-treated group. The Kyoto Encyclopedia of Genes and Genomes analysis revealed enrichment of multiple significant pathways, including those involved in necroptosis and cytokine-cytokine receptor interactions. This is the first study to demonstrate the protective effect of USCs on bladder function and remodeling in pBOO rats. The miRNA and mRNA expression levels differed in the bladder of pBOO rats with and without USC treatment. Although the mechanism underlying these effects has not been fully elucidated, necroptosis and cytokine-cytokine receptor interaction-related pathways may be involved.

\section{Background}

Partial bladder outlet obstruction (pBOO) is defined as a common urological disorder in which a partial obstruction of any part between the bladder outlet and external urethra is observed. The most common causes of pBOO are benign prostate hyperplasia in elderly men, posterior urethral valves in newborns, pelvic prolapse in women, and urethral stricture caused by iatrogenic injury. In patients with moderate or severe long-term pBOO, an increase in bladder outlet resistance pressure and intravesical pressure results in bladder tissue inflammation, detrusor muscle hypertrophy, connective tissue accumulation in the bladder wall, and tissue remodeling and decreased bladder compliance (1-4). Even after the obstruction is relieved, bladder dysfunction can persist. Symptoms such as waiting for urine, weak urination, frequent urination, and nocturia may remain or even worsen.

Owing to their self-renewal ability, multidirectional differentiation, and proliferation, stem cells have demonstrated tissue repair capabilities during the remodeling and fibrosis in multiple organ systems. Studies have confirmed that mesenchymal stem cells can inhibit the inflammatory response and fibrosis process of bladder tissue after the development of pBOO, thereby improving bladder function (5). Urinederived stem cells (USCs) are recognized as pluripotent stem cells with multidirectional differentiation potential. USCs can be directly extracted from urine more easily than extracting mesenchymal stem cells, and the process is therefore convenient and economical and does not require invasive surgery. For 
patients without kidney disease, USCs can be extracted directly from the urine, and this demonstrates a lower risk of occurrence of autoimmune reactions and involves minor ethical issues.

In this study, we used human USCs in a pBOO rat model with an aim to clarify the effect of USCs on bladder function by observing bladder urodynamics and histological changes, and investigated the microRNA (miRNA) and mRNA expression profiles of bladder smooth muscle tissue using highthroughput sequencing. We used bioinformatics methods to clarify the potential molecular pathways of USCs acting on bladder tissue.

\section{Methods}

\section{Ethics statement}

The experiments were approved by the Animal Care and Use Committee of Shenzhen University General Hospital and complied with the Guide for the Care and Use of Laboratory Animals, Eighth Edition (2011). Written informed consent was obtained from all donors prior to the collection of urine samples.

\section{Animals and study design}

The eighteen adult male Sprague-Dawley rats used in this study were purchased from the Center for Experimental Animals of Guangzhou University of Chinese Medicine. All rats weighed 190-210 $\mathrm{g}$ and were housed at $20-26{ }^{\circ} \mathrm{C}$ under a standard $12 \mathrm{~h} / 12 \mathrm{~h}$ light-dark cycle. The rats were randomly and evenly assigned to the following three groups: a sham surgery group (control group), a pBOO without USC therapy group (pBOO group), and pBOO with USC therapy group ( $\mathrm{pBOO+USCs} \mathrm{group).}$

\section{Induction of the pBOO model}

The rats in the $\mathrm{pBOO}$ and $\mathrm{pBOO+USCs}$ groups were subjected to urethral ligation under anesthesia using isoflurane to establish the pBOO model, as per methods described previously [4]. Briefly, with the rat placed in the supine position, a midline suprapubic incision was inflicted, and the bladder neck and urethra were exposed. A 1.0-mm inner diameter catheter was placed around the proximal urethra, and a 40 silk ligature was tied around the urethra and tube. After checking for tightness, the tube was removed, and the incision was closed. Six rats in the control group were subjected to the same operation to expose the bladder without urethral ligation.

\section{Isolation and culture of USCs}

USCs were collected from the urine of five healthy male donors aged 19-25 years. The collection, isolation, and identification methods have been described in our previous study [6]. Briefly, urine samples were centrifuged at $500 \times g$ for 5 min to collect the cells. The cell pellet was gently resuspended in a mixed medium consisting of embryo fibroblast medium and keratinocyte serum-free medium (1:1 ratio), following which the cells were seeded in 24 -well plates (passage $(P)$ ). Individual USCs were observed $3-5$ days after the initial seeding and reached $70 \%-80 \%$ confluency after another $3-4$ days. The cells 
were trypsinized and transferred to 6-well plates (P1). Finally, the cells were transferred to a 100-mm culture dish (P2) for expansion. P3 USCs were used in the experiments.

\section{Treatment with USCs}

The pBOO model was successfully established via urodynamic analysis seven weeks after performance of the urethral ligation procedure. Hence, all rats in the pBOO+USCs group were administered with a tail vein injection of USCs ( $2 \times 10^{6}$ cells suspended in $0.2 \mathrm{~mL}$ phosphate-buffered saline [PBS]) six times every other week. Rats in the pBOO group received an injection of an equal volume of PBS using the same process.

\section{Urodynamics}

Urodynamic studies were performed at the $7^{\text {th }}$ week post-urethral ligation to determine the baseline urodynamic values, and urethral ligation was released for rats in the pBOO groups. All rats were subjected to urodynamic studies $7,11,15$, and 19 weeks after urethral ligation. Under subjection to isoflurane anesthesia through an abdominal incision, a 27-gauge double lumen catheter was inserted into the bladder and connected to a pressure transducer (MLT488, ADinstuments, Australia). The bladder was emptied using a syringe and the abdomen was covered with a warm gauze. Saline solution was infused through the catheter at a constant rate of $0.3 \mathrm{~mL} / \mathrm{min}$. The following parameters were recorded: endfilling pressure, voided volume, maximal voiding pressure, bladder capacity, and residual volume. Bladder volume change $(\Delta V)$ was calculated as the perfusion volume from infusion to urine leakage, and bladder pressure change $(\Delta \mathrm{P})$ was calculated as the bladder leak point pressure minus the pressure before bladder perfusion. Bladder compliance (compliance, $\mathrm{C}$ ) was calculated using the formula $\Delta V / \Delta P$.

\section{Measurement of bladder muscle strip tension}

At the end of the experiments, all rats were euthanized via subjection to an isoflurane anesthetic overdose. The bladder was rapidly removed and subjected to fixation in Krebs-Henseleit solution at $4{ }^{\circ} \mathrm{C}$. The mucosa was carefully removed under a microscope and excised into longitudinal muscle strips (8 2 $\mathrm{mm}$ ) using a double-edged knife. All muscle strips were transferred to a 10-mL organ bath filled with Krebs-Henseleit solution. The bath was maintained at $37^{\circ} \mathrm{C}$ under an $95 \% \mathrm{O}_{2}$ and $5 \% \mathrm{CO}_{2}$ atmosphere. After warming for 30 min without tension, one end of each strip was ligated to a force transducer (MLT488, ADinstruments, Australia), and the other end was fixed to the bottom of the fixing plate with a 40 silk ligature. The force transducer-linked tension sensor was connected to a computer to detect and record the signal using the Powerlab multichannel biological signal analysis system (MI224, ADinstruments, Australia). The fine-tuning spiral was adjusted to slowly pull the muscle strip to increase the tension of the muscle strip. Contraction was quantified by measuring the force generated in millinewton $(\mathrm{mN})(\mathrm{g}=9.81)$. The basic value of tension was $0.7 \mathrm{mN}$, which was maintained for $15 \mathrm{~min}$. After the muscle strips were balanced, $10^{-4} \mathrm{M}$ carbachol was added to stimulate the muscle strips, and the maximum contraction value of the muscle strips was recorded. 


\section{Histological examination}

Half of the bladder was snap-frozen in liquid nitrogen for the conduction of microarray expression profiling and bioinformatics analysis. The remaining tissues were subjected to fixation in $4 \%$ paraformaldehyde and stained paraffin-embedded before analysis. The sections were stained with hematoxylin-eosin (H\&E) to observe the general morphology of the bladder, and Masson's trichrome staining was performed to evaluate the extent of tissue fibrosis. All sections were evaluated under a light microscope (Olympus, Tokyo, Japan).

\section{TUNEL assay}

A one-step TUNEL apoptosis assay kit (Beyotime Biotechnology, Shanghai, China) was used to evaluate apoptosis levels in the bladder smooth muscle. Briefly, the sections were regularly hydrated and immersed in citric acid buffer $(0.01 \mathrm{mM}, \mathrm{pH}=6)$, followed by incubation with $10 \%$ bovine serum albumin for $30 \mathrm{~min}$. Consequently, the sections were subjected to the TUNEL reaction solution for $20 \mathrm{~min}$ and then with DAPI for 10 min in a dark chamber. Finally, after regular dehydration and transparency, the sections were observed under a fluorescence microscope (Olympus, Tokyo, Japan) and analyzed by a pathologist in a blinded manner.

\section{RNA sequencing}

At the end of the experiment, total RNA extraction was performed from a section of the bladder tissue obtained from two rats in the control group, two rats in the pBOO group, and four rats in the pBOO+USCs group, and the samples were processed for RNA sequencing. A total of $1 \mu \mathrm{g}$ RNA per sample was used as the input material for the RNA sample preparation. Sequencing libraries were generated using the rRNAdepleted RNA and NEBNext ${ }^{\circledR}$ Ultra ${ }^{\mathrm{TM}}$ Directional RNA Library Prep Kit for Illumina ${ }^{\circledR}$ (NEB, USA), following the manufacturer's recommendations. The library integrity was assessed using the Agilent Bioanalyzer 2100 system (Agilent, USA). Clustering of the index-coded samples was performed using the cBot Cluster Generation System and TruSeq PE Cluster Kit v3-cBot-HS (Illumina), according to the manufacturer's instructions. After the completion of the cluster generation, the libraries were sequenced using the Illumina Hiseq 3000 platform, and 150-bp paired-end reads were generated. Reference genome and gene model annotation files were downloaded directly from the Genome website. The mapped reads of each sample were assembled using both Scripture (beta2) and Cufflinks (v2.1.1). Raw read counts were then used as input to DESeq to calculate the normalized signal for each transcript in the samples. The $p$ value was adjusted using the $q$ value. A q value $<0.01$ and $\mid \log 2$ (fold change) $\mid>1$ were set as the thresholds for significantly differential expression. For small RNA sequencing, mapping was performed using the reference sequence and Bowtie [7]. miRBase20.0 was used as a reference [8]. The target gene of miRNA was predicted using psRobot_tar in miRanda for animals [9]. In the present study, FUNRICH (http://www.funrich.org/) was used to perform Gene Ontology (GO), while the Kyoto Encyclopedia of Genes and Genomes (KEGG) was used for pathway analyses.

\section{Statistical analysis}


All values are presented as mean SD. Statistical analyses were performed using SPSS v.22.0 (SPSS Inc., Chicago, IL, USA). The Student-Newman-Keuls (SNK) analysis of variance was performed to evaluate the differences among the rat groups considered herein. Statistical significance was set at $p<0.05$.

\section{Results}

\section{Urodynamics}

Urodynamic studies in all groups reveal the highest bladder compliance in controls and the lowest compliance in both pBOO groups in the 7th week post-urethral ligation (48.57 \pm 8.61 vs. $21.00 \pm 3.87$ $\mu \mathrm{L} / \mathrm{cm} \mathrm{H}_{2} \mathrm{O}$, respectively, $p<0.01$ ). After subjection to two injections of USCs, compliance gradually improves in the $\mathrm{pBOO}+\mathrm{USCs}$ group in the 15th week compared with that in the pBO0 group (38.35 \pm 2.81 vs. $16.57 \pm 4.69 \mu \mathrm{L} / \mathrm{cm} \mathrm{H}_{2} \mathrm{O}$, respectively, $p<0.01$ ), and is similar to that of the controls in the 19th week (48.50 \pm 7.28 vs. $50.07 \pm 8.04 \mu \mathrm{L} / \mathrm{cm} \mathrm{H}_{2} \mathrm{O}$, respectively, $p=0.89$ ) (Fig. 1A). The maximal voiding pressure in the pBOO group is significantly lower than that in the control group in the 7th week $(24.32 \pm$ 3.22 vs. $46.80 \pm 10.17 \mathrm{~cm} \mathrm{H}_{2} \mathrm{O}$, respectively, $p<0.01$ ), whereas $\mathrm{pBOO+USCs}$ gradually presents with an increasing pressure from the 15th week $\left(38.31 \pm 5.21\right.$ vs. $22.61 \pm 2.60 \mathrm{~cm} \mathrm{H}_{2} \mathrm{O}$, respectively, $\left.p<0.01\right)$ (Fig. 1B). End-filling pressure in pBOO rats is higher than that in controls $(10.96 \pm 2.07$ vs. $6.84 \pm 1.80$ $\mathrm{cm} \mathrm{H}_{2} \mathrm{O}$, respectively, $\left.p=0.006\right)$, and it increases from the 7th to 19th week $(10.96 \pm 2.07$ in 7th week $v s$. $16.15 \pm 1.68 \mathrm{~cm} \mathrm{H}_{2} \mathrm{O}$ in 19th week, $p<0.01$ ), whereas the pressure in $\mathrm{pBOO}+$ USCs rats decreases to the control level ( $9.03 \pm 0.99$ vs. $7.26 \pm 1.94 \mathrm{~cm} \mathrm{H}_{2} \mathrm{O}$, respectively, $\left.p=0.359\right)$ in the 15 th week (Fig. $\left.1 \mathrm{C}\right)$. All $\mathrm{pBOO}$ and $\mathrm{pBOO}+\mathrm{USC}$ rats demonstrate significantly higher residual volume than that in control rats $(0.70 \pm 0.11$ vs. $1.36 \pm 0.19 \mathrm{~mL}$, respectively, in the 7th week $[p<0.01])$; however, USCs considerably suppresses the upward trend from the 15th week $(1.26 \pm 0.12 \mathrm{vs} .1 .93 \pm 0.10 \mathrm{~mL}, p<0.01)$ (Fig. 1D). Moreover, pBOO rats exhibit lower voided volume than that in control rats in the 7th week $(238.77 \pm$ $27.11 v s .337 .84 \pm 21.49 \mu \mathrm{L}, p<0.01$ ); however, the pBO0+USCs group exhibits an increasing trend and finally demonstrates similar voided volume as the control group in the 19th week (340.75 $\pm 16.93 \mathrm{vs}$. $316.51 \pm 16.23 \mu \mathrm{L}$, respectively, $p=0.119$ ) (Fig. 1E). Additionally, a significant increase in the bladder capacity is observed in all pBOO rats compared with that in the control $(1.54 \pm 0.14$ vs. $1.04 \pm 0.14 \mathrm{~mL}$, respectively, $p<0.01$ ). Although USCs reversed the trend in the pBO0+USCs group from the 15th week $(1.59 \pm 0.23$ vs. $2.17 \pm 0.17 \mathrm{~mL}, p<0.01)$, the bladder capacity does not decrease to the level of the control (1.52 \pm 0.27 vs. $1.08 \pm 0.16 \mathrm{~mL}$, respectively, $p<0.01)$ (Fig. 1F). Representative cystometrograms of three rat groups at 7, 11, 15, and 19 weeks after urethral ligation are depicted in Fig. 1G.

\section{Histology and TUNEL assay}

H\&E staining revealed that the bladder wall thickness is higher, the urothelium flatter, and structural damage of detrusor smooth muscle worse in the pBOO group than those observed in the control group; however, treatment with USCs significantly alleviate these histological changes (Fig. 2A). Masson's 
trichrome staining revealed the presence of increased collagen content, disorderly and loosely arranged detrusor muscle bundles, and an evidently widened gap between muscle bundles in the pBOO group compared with that in the control group and pBOO + USCs group (Fig. 2B). There is a marked increase in the number of TUNEL-positive cells in the $\mathrm{pBOO}$ group compared with that in the control group, whereas USC treatment significantly decreases the number of apoptotic cells in the bladder (Fig. 2C).

\section{Assessment of detrusor muscle contractility}

In the detrusor tension tests, the bladder detrusor contraction amplitudes of pBOO rats were significantly lower than those of control rats. Carbochol elevated the contraction amplitudes at different

concentrations $\left(10^{-6}\right.$ to $\left.10^{-4} \mathrm{M}\right)$ in the three groups, but the bladder detrusor of $\mathrm{pBOO}$ rats exhibited the lowest sensitivity to carbochol. The effect of carbochol on the amplitudes of contraction in pBOO + USCs rats is significantly higher than that in $\mathrm{pBOO}$ rats $(4.58 \pm 0.89 \mathrm{vs.} 2.85 \pm 0.23 \mathrm{mM}$, respectively, $p<0.01)$; however, it is lower than that in control rats $(4.58 \pm 0.89$ vs. $6.04 \pm 0.26 \mathrm{mM}$, respectively, $p<0.01)$ (Fig. 2D).

\section{Bioinformatics analysis of miRNA-mRNA}

Bioinformatics analysis revealed differentially expressed genes (DEGs) and differentially expressed miRNAs (DEMs) in the bladder wall tissue among the control, pBOO, and pBOO + USCs groups in rats. Below, we have provided the results for miRNAs and mRNAs that were regulated in the pBOO and USC treatment relative to the controls. The comparisons are therefore $\mathrm{pBOO}$ relative to the control group and $\mathrm{pBOO}+$ USCs relative to the $\mathrm{pBOO}$ group.

The volcano plots in Fig. $3 a$ and $b$ show DEMs in rat bladder wall tissues of the control, pBOO, and pBOO + USCs groups. The distribution of all miRNAs with respect to significance ( $y$-axis) vs. foldchange ( $x$-axis) has been illustrated using the volcano plot. Compared with the control group, the present study helps identify 62 DEMs (40 upregulated and 22 downregulated) and 1686 DEGs (981 upregulated and 705 downregulated) in the pBOO group. Compared with the pBOO group, we identify 42 DEMs (25 upregulated and 17 downregulated) and 757 DEGs (241 upregulated and 516 downregulated) in the pBOO + USCs group. The expression of eight DEMs, which are upregulated in $\mathrm{pBOO}$ rats relative to control rats, is reversed via USC treatment (Fig. 3).

Five of the eight DEMs, namely miR-142-3p, miR-142-5p, miR-187-3p, miR-503-5p, and 17_19667_star, present upregulated expression in $\mathrm{pBOO}$ rats (Fig. $3 \mathrm{~A}$ ) and the expression level is downregulated by USC treatment (Fig. 3B). The Venn diagrams illustrating downregulated mRNAs expressed in rats from pBOO $v s$. control group (pBOO vs. ctrl mRNA_lo) and upregulated mRNAs expressed in rats from pBOO + USCs vs. pBOO group (pBOO + USCs vs. pBOO mRNA_up), and the target genes of the above-mentioned five DEMs, help identify three DEGs (Fig. 3). 
Three of the eight DEMs, namely miR-9a-3p, miR-9a-5p, and 1_1401, exhibit downregulated expression in pBOO rats (Fig. 3A) and upregulated expression via USC treatment (Fig. 3B). Similarly, the Venn diagrams illustrating upregulated mRNAs expressed in rats from $\mathrm{pBOO} v s$. control group (pBOO vs. ctrl mRNA_up) and downregulated mRNAs expressed in rats from pBOO + USCs vs. pBOO group (pBOO + USCs vs. pBOO mRNA_lo), and the target genes of the above-mentioned three DEMs, help identify 17 DEGs (Fig. 3). The changes in the levels of these DEMs in the three groups are shown in the gene trend map in Fig. $3 \mathrm{C}$ and D.

Following the above-mentioned analysis, a miRNA-gene interaction network was constructed by integrating data on the target genes with DEGs. As shown in Fig. 4A, the pBOO and USC-associated DEM-DEG interaction network was constructed, which comprised 23 nodes, including four that were DEMs, and 22 connections. miR-9a-3p and miR-9a-5p were determined as the two largest nodes of the four DEMs and established interactions with 13 and five DEGs, respectively.

$\mathrm{GO}$ analysis reveal that the most significantly enriched GO terms that correspond to the above-mentioned target DEGs were "superoxide-generating NADPH oxidase activity" (ontology: MF), "NADPH oxidase complex" (ontology: $\mathrm{CC}$ ), and "semaphorin-plexin signaling pathway involved in axon guidance" (ontology: BP) (Fig. 4B).

Furthermore, KEGG analysis revealed that multiple significant pathways are enriched, which are primarily associated with leukocyte transendothelial migration, chemokine signaling pathway, cytokine-cytokine receptor interaction, necroptosis, nucleotide-binding and oligomerization domain (NOD)-like receptor signaling pathway, and apoptosis signaling pathway (Fig. 4C).

\section{Discussion}

PBOO initiates a pathophysiological cascade of events in the bladder wall, including inflammation, hypoxia, collagen deposition, smooth muscle hypertrophy, apoptosis, and fibrosis. The obstructed bladder undergoes modification with respect to its structure to compensate for the increased resistance to flow, and significant hypoxia ensues owing to the development of high resistance to flow and consequent high intravesical pressure (1-4). Studies in 2 week-obstructed rabbit models revealed the existence of a larger capacity and lower intravesical pressure and compliance of the bladder $(2,4)$. Additionally, studies in Sprague-Dawley rats have shown an increase in end-filling pressure, residual volume, and bladder capacity, as well as a decrease in maximal voiding pressure and bladder compliance $(2,10)$. Some parameters such as voided volume vary with the duration of obstruction time. Yuan et al. revealed that voided volume increased in 3 week-obstructed rats and decreased in 6 week-obstructed group (3). Bladder strip contractility tension and tension sensitivity reportedly decrease in various animal models, including mice, rats, and rabbits $(4,10,11)$.

Presently, there exists a lack of availability of effective treatments for the improvement of bladder function after long-term moderate to severe pBOO in clinical practice. Studies have confirmed the effect of MSCs on the bladder function of pBOO animal models based on the potential applicability of stem cells in tissue reconstruction and organ repair engineering $(5,12-14)$. However, the mechanism has not 
been explored at the genetic and molecular levels. Additionally, the disadvantages of MSCs, including the invasive extraction process and high cost, have limited their future clinical applications. Compared with MSCs, USCs are deemed advantageous owing to the simple, noninvasive, and low-cost harvesting methods. Studies have revealed the nephron-protective effect of USCs on renal function realized via antiinflammatory, antioxidative stress, and antifibrotic activities in acute and chronic kidney disease $(15,16)$. Moreover, USC-exosomes have exhibited pro-neurogenesis effects in the rat brain after the occurrence of ischemic stroke and have demonstrated the exertion of pro-angiogenesis effects and improvement in erectile dysfunction in diabetic rats, thereby exhibiting bladder protection functions (17-19). Presently, there are no reports available on the effects of USCs on the bladder function in patients with pBOO.

With the aim to explore the effect of USCs on bladder function and morphology in moderate or severe pBOO, we designed a long-term pBOO rat model and dynamically observed the changes from 7- to 19week-obstructed rats. Similar to the findings reported in previous studies, we observed elevated end-filling pressure, residual volume, and bladder capacity and declined maximal voiding pressure, voided volume, and bladder compliance in the pBOO group compared with the control group. Additionally, once a lowcompliance bladder was established, cystometric parameters did not improve without intervention, even if the urethral ligature was removed. Certain parameters, including end-filling pressure, residual volume, and bladder capacity, consistently increased after ligature removal, which consequently resulted in worsening of bladder function. Similar to the case observed in most patients with moderate to severe long-term prostatic hyperplasia, the lower urinary tract symptoms of patients with pBOO cannot be relieved even if there is acceptance of resection of the prostate to relieve bladder outlet obstruction. After subjection to treatment with USCs, few cystometric parameters were significantly improved, including elevated bladder compliance and maximal voiding pressure, declined end-filling pressure, and voided volume. Although residual volume and bladder capacity did not show recovery, there was no further deterioration after subjection to USC treatment compared with the pBOO group. The detrusor muscle contractility and carbachol sensitivity were also improved after USC treatment. Results from the histological analysis and TUNEL assay revealed that the improvement of these functions was mainly related to the reduction of collagen deposition in the extracellular matrix and lowered muscle cell apoptosis.

Furthermore, the urinary bladder was exposed to mechanical stress and was subjected to treatment with USCs, which undoubtedly resulted in modification of gene and protein expression profiles in epithelial and smooth muscle cells, consequently altering the ultrastructure and physiology of cells in the bladder wall. To elucidate the molecular mechanisms underlying this complex process, we conducted bioinformatics analysis of miRNA-mRNA expression profiles of rats in the three groups. We focused on the numerous miRNAs and mRNAs that were significantly and differentially expressed in the bladder following pBOO; those that underwent reversed expression following USC treatment were examined. We identified miR-142 and miR-9a as the two largest nodes of DEMs in the miRNA-gene interaction network.

MiR-142-3p and miR-142-5p are reportedly overexpressed in the bladder tissue of pBO0 mice (20). A previous study confirmed that miR-142-5p is overexpressed in the bladder of patients with pBOO (21). MiR-142-3p and miR-142-5p influence inflammation and immune response, the levels of which have been

Page $10 / 19$ 
shown to decrease in mouse models of cardiac hypertrophy. miR-142-5p targets acetyltransferase p300 and is implicated in cardiac growth; additionally, an increase in miR-142 expression during cardiac growth is critical for cell survival $(22,23)$. The in vivo inhibition of miR-142-5p with locked nucleic acid-modified oligonucleotides reduces CCL4-induced liver fibrosis and bleomycin-induced lung fibrosis in mice. Furthermore, macrophages derived from the tissue samples of patients with liver cirrhosis and idiopathic pulmonary fibrosis display increased levels of miR-142-5p (24). Therefore, it can be inferred that miR-142$5 p$ regulates macrophage profibrogenic gene expression during chronic inflammation. In this study, miR142-3p and miR-142-5p were overexpressed in pBOO rats, whereas their expression was suppressed by USCs, indicating that miR-142 participated in the antifibrosis effect of USCs in pBOO rats.

Studies have revealed that miR-9a participates in anti-inflammatory and anti-apoptotic functions and is related to neurogenesis. The downregulation of miR-9a-5p expression may increase the levels of NLRP1 inflammasome proteins, cleaved caspase-1, interleukin (IL)-1 b, and IL-18. In contrast, the overexpression of miR-9a-5p markedly prevents the abnormal expression of these proteins and is accompanied by the downregulation of NLRP1 expression in ischemia-like conditions, both in vivo and in vitro (25). Further, miR-9 prevents cardiac dysfunction and inhibits cardiomyocyte apoptosis in myocardial infarction mouse hearts (26). However, a study indicated that miR-9a-5p is a novel regulator of HSCs in the setting of increased pressure and hepatic fibrosis in rats and that the overexpression of miR-9a-5p following hepatic stellate cell activation perpetuates the fibrotic response (27). In our study, we found that both miR-9a-3p and miR-9a-5p levels were decreased in pBOO rats and increased in USC-treated pBOO rats. They may confer protection to bladder functions by reducing the inflammatory response of bladder tissue and reducing detrusor cell apoptosis, aspects which should be confirmed through further studies.

To obtain insights into the classification of miRNAs, we conducted GO and KEGG analyses to predict the target genes and possible pathways. The results revealed various $\mathrm{GO}$ terms associated with superoxidegenerating NADPH oxidase activity, NADPH oxidase complex, semaphorin receptor activity, semaphorinplexin signaling pathway involved in axon guidance, oxidoreductase activity, acting on $\mathrm{NAD}(\mathrm{P}) \mathrm{H}$, oxygen as an acceptor, and hydrogen peroxide biosynthetic process. Additionally, it was observed that various pathways were involved, including leukocyte transendothelial migration, chemokine signaling pathway, cytokine-cytokine receptor interaction, necroptosis, NOD-like receptor signaling pathway, and apoptosis. The necroptosis and cytokine-cytokine receptor interaction signaling pathways may be responsible for the pathological alterations observed in this study and may help provide insights for the formulation of a strategy for therapeutic intervention, following further investigation. The necroptosis signaling pathway is a programmed form of necrosis. It can be initiated by different stimuli, such as tumor necrosis factor (TNF) and TNF-related apoptosis-inducing ligand. It has previously been demonstrated that cytokine and immune response pathways, transforming growth factor, nitric oxide signaling, and hypertrophic $\mathrm{PI} 3 \mathrm{~K} / \mathrm{AKT}$ signaling pathways are shared among all pBOO phenotypes. AP-1 and NF-kB are considered the dominant transcription factors, and TNF-a is recognized as the top upstream regulator (21). However, classic fibrosis-related pathways such as the transforming growth factor beta signaling pathway were not identified in this analysis. Therefore, it may be implied that USCs may inhibit the initiation of the pathophysiological cascade of events in the bladder wall via the necroptosis and cytokine-cytokine 
receptor interaction signaling pathways. Additional research is warranted to verify the exact roles of the identified miRNAs, genes, and signaling pathways in the pathogenesis of bladder remodeling induced by pBOO.

\section{Conclusions}

To the best of our knowledge, this study is the first to identify the protective effects of USCs on bladder function and remodeling in pBOO rats. Treatment with USCs elevated bladder compliance and maximal voiding pressure, reduced end-filling pressure and voided volume, strengthened detrusor muscle contractility, and increased sensitivity to carbachol. These alterations were associated with a reduction in collagen deposition in the extracellular matrix and a reduction in cell apoptosis. Alterations in miRNA and mRNA expression levels in the bladder of pBOO rats with and without USC treatment were observed. Bioinformatics analysis of these alterations may help elucidate and improve our understanding of the molecular mechanisms underlying the effects of USCs in pBOO rats. Both miR-142 and miR-9a may play regulatory roles via anti-inflammatory, anti-apoptotic, and antifibrotic effects. Although the mechanisms by which these effects occur have not been fully elucidated, it may be suggested that USCs exert their benefits via necroptosis and cytokine-cytokine receptor interaction signaling pathways. Further studies are therefore necessary to verify these initial outcomes on the potential applicability of USC-mediated bladder repair strategies in the setting of obstructive injury.

\section{Abbreviations}

$\mathrm{DEG}=$ differentially expressed gene

DEM $=$ differentially expressed miRNA

$\mathrm{GO}=$ Gene Ontology

H\&E = hematoxylin-eosin

KEGG $=$ Kyoto Encyclopedia of Genes and Genomes

pBOO $=$ partial bladder outlet obstruction

PBS $=$ phosphate-buffered saline

TNF = tumor necrosis factor

USCs = urine-derived stem cells

\section{Declarations}

Ethics approval and consent to participate 
The experiments were approved by the Animal Care and Use Committee of Shenzhen University General Hospital and complied with the Guide for the Care and Use of Laboratory Animals, Eighth Edition (2011). Written informed consent was obtained from all donors prior to the collection of urine samples.

Consent for publication

Not applicable.

Availability of data and materials

The datasets generated and/or analyzed in the current study are available in the GEP repository (accession No. GSE171367).

Competing interests

The authors declare that they have no competing interests.

Funding

This study was sponsored by the Science and Technology Innovation Commission Foundation of Shenzhen (JCYJ20190808141013454 and JCYJ20180305124827261)『and Shenzhen Key Laboratory Foundation (ZDSYS20200811143757022).

Authors' contributions

MT designed the methods and was a major contributor in the preparation of the manuscript. RW and PZ contributed to the animal model establishment and conducted the animal experiments. QW and BS contributed to the isolation and culture of USCs. KL conducted the urodynamic studies. JZ analyzed and interpreted the bioinformatics data. WX and HG analyzed and interpreted the urodynamic data. SL contributed to the histological examination. YW and XW made substantial contributions to the conception of the study and revised the manuscript. All authors read and approved the final manuscript.

Acknowledgements

The authors would like to acknowledge all volunteers who donate their urine samples for USCs collection. We also acknowledge the funding support from the Science and Technology Innovation Commission Foundation of Shenzhen (JCYJ20190808141013454 and JCYJ20180305124827261)『and Shenzhen Key Laboratory Foundation (ZDSYS20200811143757022).

\section{References}

1. Duan, L. J., Cao, Q. F., Xu, D., Liu, H. L., \& Qi, J. (2017). Bioinformatic analysis of microRNA-mRNA expression profiles of bladder tissue induced by bladder outlet obstruction in a rat model. Molecular Medicine Reports, 16, 4803-4810 
2. Malkowicz, S. B., Wein, A. J., Elbadawi, A., Van Arsdalen, K., Ruggieri, M. R., \& Levin, R. M. (1986). Acute biochemical and functional alterations in the partially obstructed rabbit urinary bladder. Journal of Urology, 136, 1324-1329

3. Yuan, X., Wu, S., Lin, T., He, D., Li, X., Liu, S. ... Guanghui, W. (2011). Role of nitric oxide synthase in bladder pathologic remodeling and dysfunction resulting from partial outlet obstruction. Urology, 77, 10081-10088

4. Oka, M., Fukui, T., Ueda, M., Tagaya, M., Oyama, T., \& Tanaka, M. (2009). Suppression of bladder oxidative stress and inflammation by a phytotherapeutic agent in a rat model of partial bladder outlet obstruction. Journal of Urology, 182, 382-390

5. Wiafe, B., Adesida, A. B., Churchill, T., Kadam, R., Carleton, J., \& Metcalfe, P. D. (2019). Mesenchymal stem cell therapy inhibited inflammatory and profibrotic pathways induced by partial bladder outlet obstruction and prevented high-pressure urine storage. Journal of Pediatric Urology, 15, 2541-2510

6. Wang, Q., Zhao, J., Wu, C., Yang, Z., Dong, X., Liu, Q. ... Li, L. (2017). Large conductance voltage and $\mathrm{Ca}(2+)$-activated $\mathrm{K}(+)$ channels affect the physiological characteristics of human urine-derived stem cells. American Journal of Translational Research, 9, 1876-1885

7. Langmead, B., Trapnell, C., Pop, M., \& Salzberg, S. L. (2009). Ultrafast and memory-efficient alignment of short DNA sequences to the human genome. Genome Biology, 10, R25

8. Friedländer, M. R., Mackowiak, S. D., Li, N., Chen, W., \& Rajewsky, N. (2012). miRDeep2 accurately identifies known and hundreds of novel microRNA genes in seven animal clades. Nucleic Acids Research, 40, 37-52

9. Enright, A. J., John, B., Gaul, U., Tuschl, T., Sander, C., \& Marks, D. S. (2003). MicroRNA targets in Drosophila. Genome Biology, 5, R1

10. Zeng, J., Xie, K., Jiang, C., Mo, J., \& Lindström, S. (2012). Bladder mechanoreceptor changes after artificial bladder outlet obstruction in the anesthetized rat. Neurology and Urodynamics, 31, 178-184

11. Austin, J. C., Chackom, S. K., DiSanto, M., Canning, D. A., \& Zderic, S. A. (2004). A male murine model of partial bladder outlet obstruction reveals changes in detrusor morphology, contractility and Myosin isoform expression. Journal of Urology, 172, 1524-1528

12. Al-Saikan, B., Ding, J., Tredget, E., \& Metcalfe, P. (2016). Benefits of mesenchymal stem cells after partial bladder outlet obstruction. Canadian Urological Association Journal, 10, E1-6

13. Woo, L. L., Tanaka, S. T., Anumanthan, G., Pope, J. C., Thomas, J. C., Adams, M. C. ... Bhowmick, N. A. (2011). Mesenchymal stem cell recruitment and improved bladder function after bladder outlet obstruction: preliminary data. Journal of Urology, 185, 1132-1138

14. Wiafe, B., Adesida, A., Churchill, T., \& Metcalfe, P. (2018). Mesenchymal stem cells inhibit hypoxiainduced inflammatory and fibrotic pathways in bladder smooth muscle cells. World Journal of Urology, 36, 1157-1165

15. Sun, B., Luo, X., Yang, C., Liu, P., Yang, Y., Dong, X. ... Li, L. (2019). Therapeutic effects of human urinederived stem cells in a rat model of cisplatin-induced acute kidney injury in vivo and in vitro.Stem Cells International, 2019,2019:8035076. 
16. Zhang, C., George, S. K., Wu, R., Thakker, P. U., Abolbashari, M., Kim, T. H. ... Atala, A. (2020). Renoprotection of urine-derived stem cells in a chronic kidney disease rat model induced by renal ischemia and nephrotoxicity. International Journal of Biological Sciences, 16, 435-446

17. Ouyang, B., Xie, Y., Zhang, C., Deng, C., Lv, L., Yao, J. ... Deng, C. (2019). Extracellular vesicles from human urine-derived stem cells ameliorate erectile dysfunction in a diabetic rat model by delivering proangiogenic microRNA. Sexual Medicine, 7, 241-250

18. Ling, X., Zhang, G., Xia, Y., Zhu, Q., Zhang, J., Li, Q. ... Deng, Z. (2020). Exosomes from human urinederived stem cells enhanced neurogenesis via miR-26a/HDAC6 axis after ischaemic stroke. Journal of Cellular and Molecular Medicine, 24, 640-654

19. Dong, X., Zhang, T., Liu, Q., Zhu, J., Zhao, J., Li, J. ... Li, L. (2016). Beneficial effects of urine-derived stem cells on fibrosis and apoptosis of myocardial, glomerular and bladder cells. Molecular and Cellular Endocrinology, 427, 21-32

20. Gheinani, A. H., Köck, I., Vasquez, E., Baumgartner, U., Bigger-Allen, A., Sack, B. S. ... Monastyrskaya, K. (2018). Concordant miRNA and mRNA expression profiles in humans and mice with bladder outlet obstruction. American Journal of Clinical and Experimental Urology, 6, 219-233

21. Gheinani, A. H., Kiss, B., Moltzahn, F., Keller, I., Bruggmann, R., Rehrauer, H. ... Monastyrskaya, K. (2017). Characterization of miRNA-regulated networks, hubs of signaling, and biomarkers in obstruction-induced bladder dysfunction. JCI Insight, 2, e89560

22. Iguchi, N., Hou, A., Koul, H. K., \& Wilcox, D. T. (2014). Partial bladder outlet obstruction in mice may cause E-cadherin repression through hypoxia induced pathway. Journal of Urology, 192, 964-972

23. Sharma, S., Liu, J., Wei, J., Yuan, H., Zhang, T., \& Bishopric, N. H. (2012). Repression of miR-142 by p300 and MAPK is required for survival signalling via gp130 during adaptive hypertrophy. EMBO Molecular Medicine, 4, 617-632

24. Su, S., Zhao, Q., He, C., Huang, D., Liu, J., Chen, F. ... Song, E. (2015). miR-142-5p and miR-130a-3p are regulated by IL-4 and IL-13 and control profibrogenic macrophage program. Nature Communications, 6,8523

25. Cao, Y., Zhang, H., Lu, X., Wang, J., Zhang, X., Sun, S. ... Cui, L. (2020). Overexpression of microRNA9a-5p ameliorates NLRP1 inflammasome-mediated ischemic injury in rats following ischemic stroke. Neuroscience, 444, 106-117

26. Yang, D., Yu, J., Liu, H. B., Yan, X. Q., Hu, J., Yu, Y. ... Du, Z-M. (2019). The long non-coding RNA TUG1miR-9a-5p axis contributes to ischemic injuries by promoting cardiomyocyte apoptosis via targeting KLF5. Cell Death \& Disease, 10, 908

27. Qi, F., Hu, J. F., Liu, B. H., Wu, C. Q., Yu, H. Y., Yao, D. K., \& Zhu, L. (2015). MiR-9a-5p regulates proliferation and migration of hepatic stellate cells under pressure through inhibition of Sirt1. World Journal of Gastroenterology, 21, 9900-9915

\section{Figures}



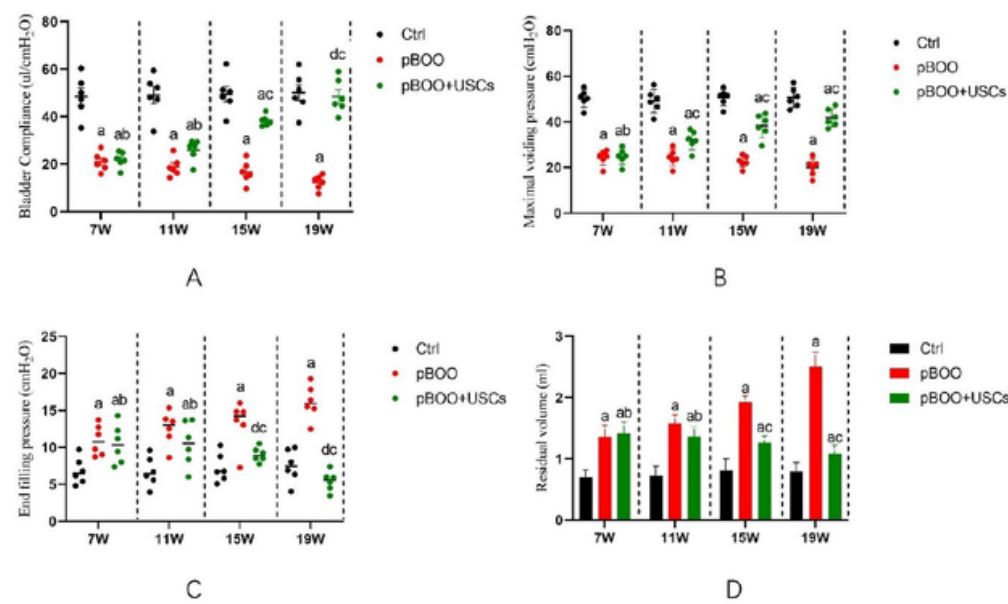

=
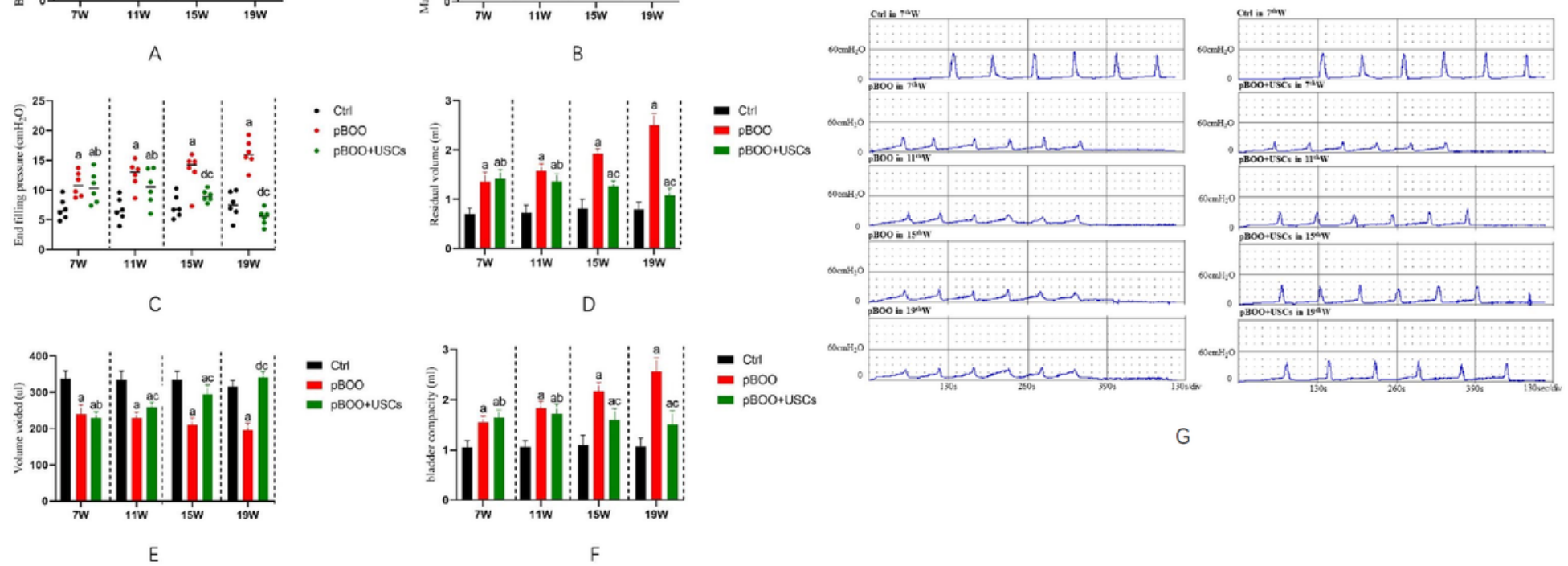

G

\section{Figure 1}

Effects of urine-derived stem cells (USCs) on bladder function. (A-F) Cystometric parameters, end-filling pressure, residual volume, and bladder capacity increased, and maximal voiding pressure, voided volume, and bladder compliance decreased in partial bladder outlet obstruction (pBOO) rats. Injection with USCs improved bladder micturition function with respect to end-filling pressure, maximal voiding pressure, and voided volume, thereby resulting in an increase in bladder compliance. a: $p<0.05$, vs. control group, b: $p$ $>0.05$, vs. $p B 00$ group, $c: p<0.05$, vs. $p B 00$ group, $d: p>0.05$, vs. control group. (G) Representative cystometrograms depicted for three rat groups at $7,11,15$, and 19 weeks after urethral ligation. 
A

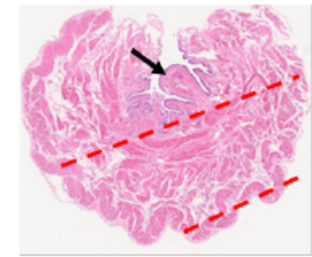

Control

B

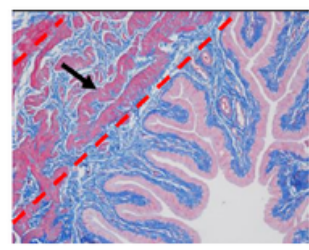

Control

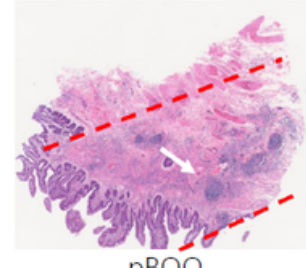

$\mathrm{pBOO}$

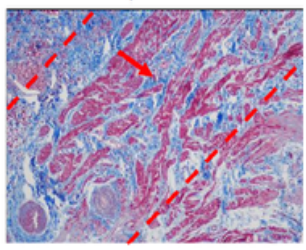

$\mathrm{pBOO}$

D

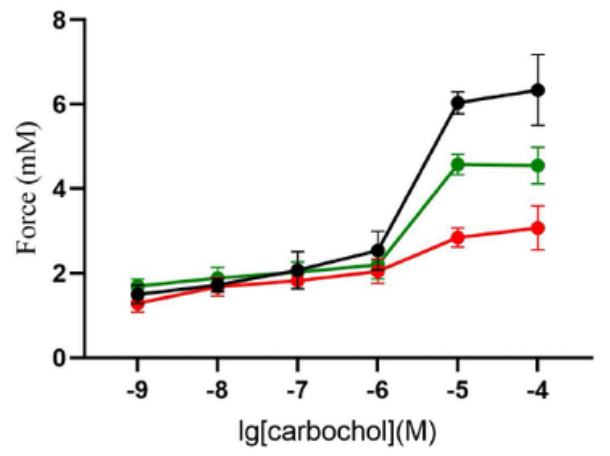

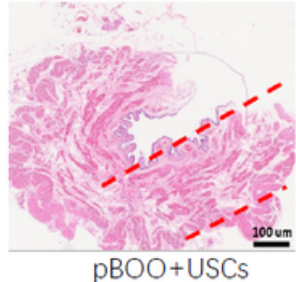

$\mathrm{pBOO}+$ USCs

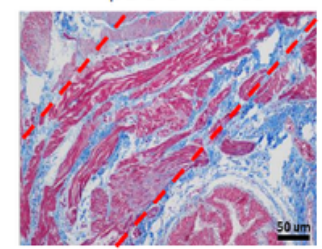

$\mathrm{pBOO+USCs}$

$\rightarrow$ Ctrl

$\rightarrow$ pBOO

$\rightarrow$ pBOO+USCs
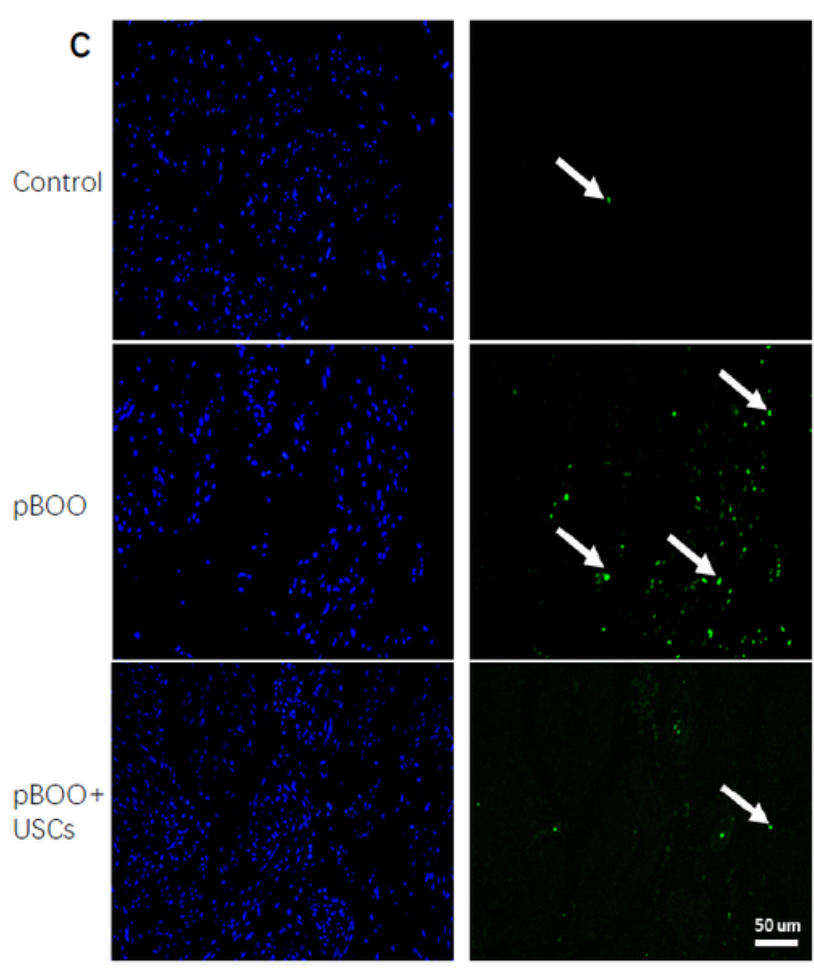

\section{Figure 2}

Changes of bladder wall histology and detrusor muscle contraction. (A-C) Hematoxylin and eosin staining, Masson's trichrome staining, and TUNEL assay revealed the deposition of a fibrous matrix and cellular apoptosis in the bladder wall, and USC treatment improved the fibrosis and cell apoptosis in the bladder wall of pBOO+USCs rats. (D) The detrusor muscle contractility, induced by carbachol, was significantly lower in pBOO rats than that in control rats. The pBOO+USCs rats demonstrated a higher sensitivity to carbachol in detrusor muscle contraction. 

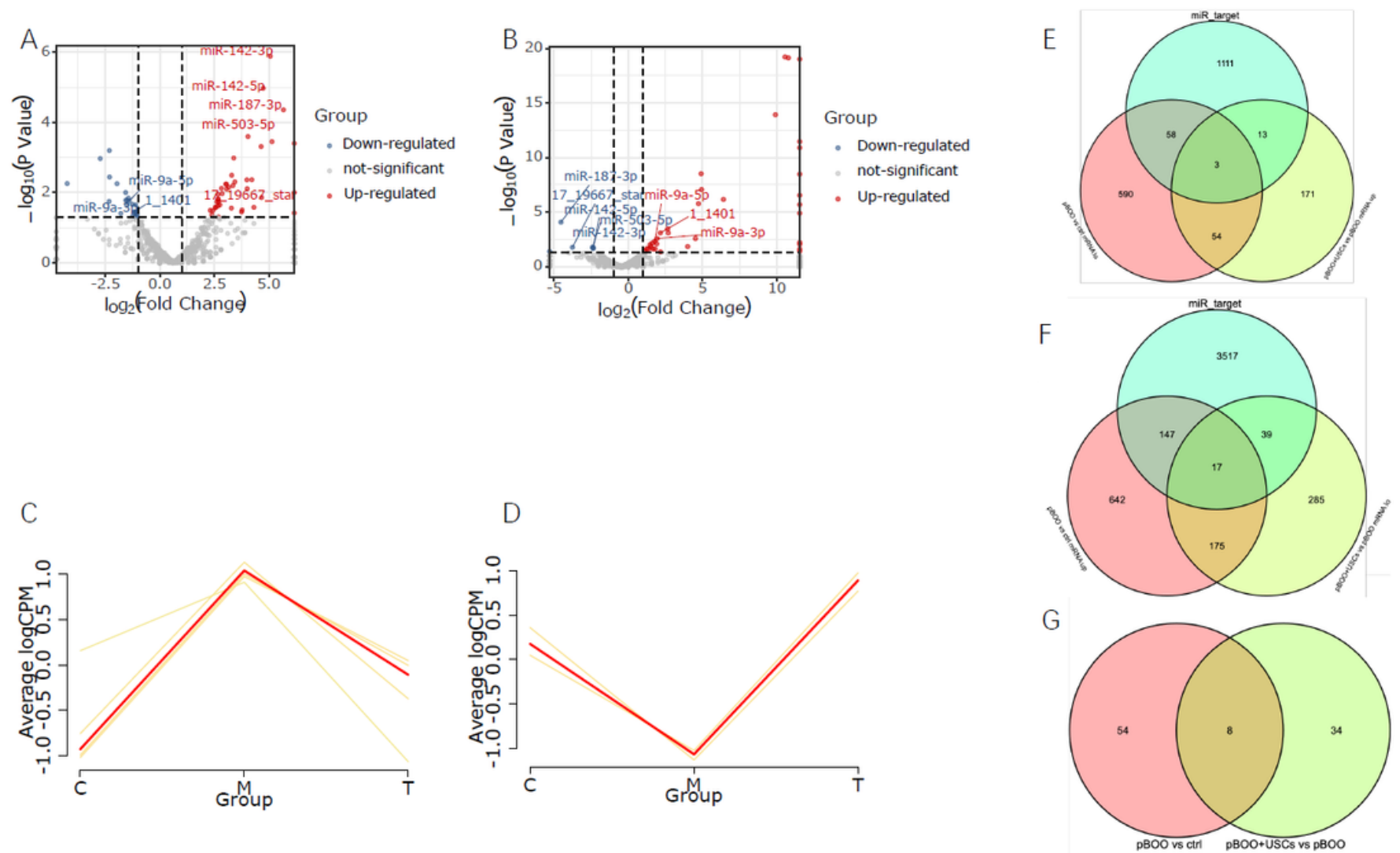

\section{Figure 3}

Differentially expressed miRNAs (DEMs) and differentially expressed genes (DEGs) changes induced by pBOO and USCs. Volcano plots illustrating DEMs in rats in the pBOO vs. control group (A) and the pBO0+USCs vs. pBOO group (B). Log2 transformation value of the fold change in miRNA expression between each group plotted on the $x$-axis. The log $p$ value (base 10) is plotted on the y-axis. DEMs (fold change $\geq 1$ ) are indicated in red (upregulated miRNAs) and blue (downregulated miRNAs). (C-D) The gene trend of DEMs in the three groups. (E) The overlapping target genes of the five DEMs and downregulated mRNAs expressed in rats from pBOO vs. control group (pBOO vs. ctrl mRNA_lo) and upregulated mRNAs expressed in rats from pBOO+USCs vs. pBOO group (pBOO+USCs vs. pBOO mRNA_up) were identified using Venn diagrams. (F) The overlapping target genes of the five DEMs and upregulated mRNAs expressed in rats from pBOO vs. control group (pBOO vs. ctrl mRNA_up) and downregulated mRNAs expressed in rats from pBOO+USCs vs. pBOO group (pBOO+USCs vs. pBOO mRNA_lo) were identified using Venn diagrams. 

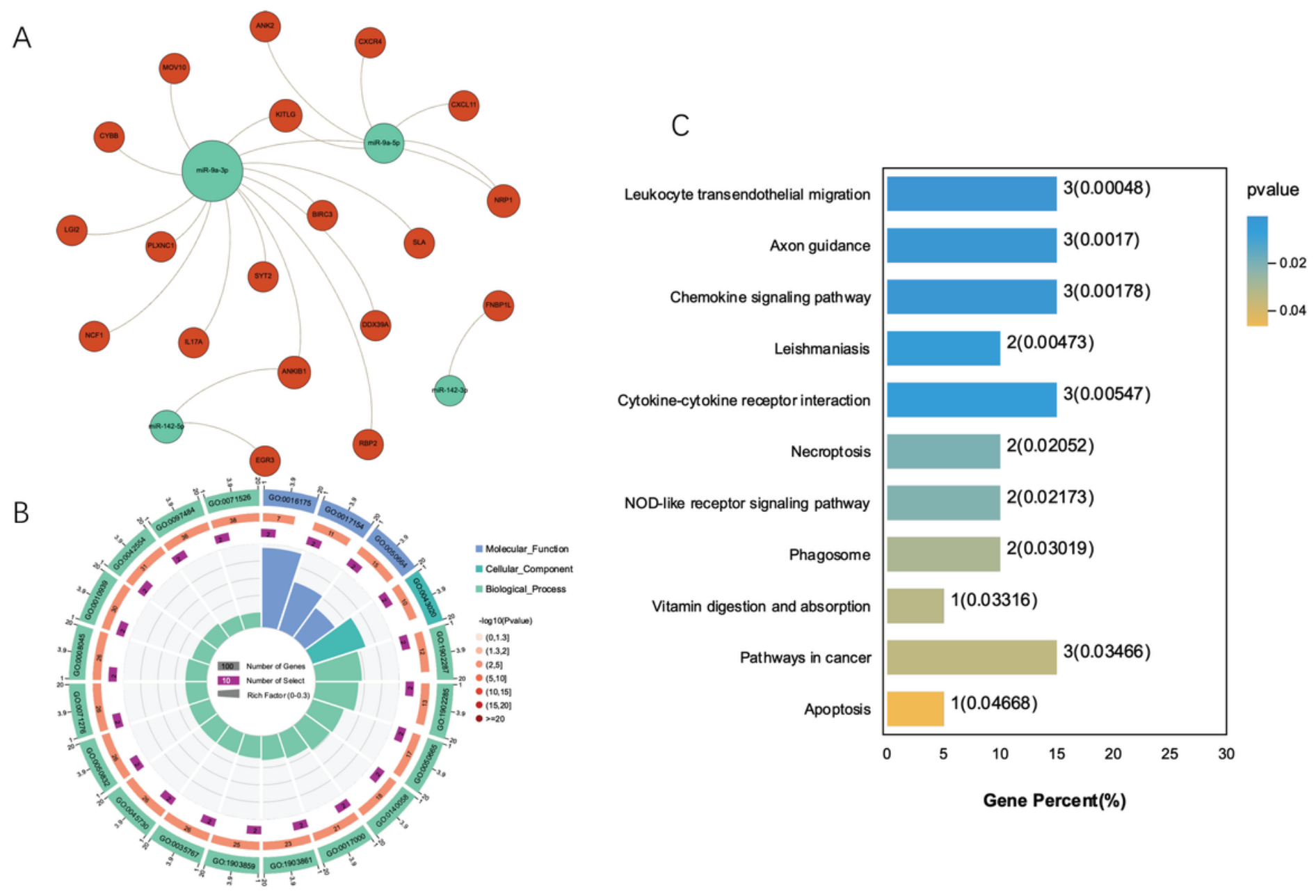

Figure 4

DEMs-DEGs regulatory network and bioinformatic analysis of bladder miRNA-mRNA. (A) Regulation networks of miRNA-mRNA were constructed using the Gephi software (version 0.9.1). Green dots represent miRNAs, whereas red dots indicate downstream target genes. (B) Enriched Gene Ontology (GO) terms for target genes. (C) Kyoto Encyclopedia of Genes and Genomes (KEGG) analysis of the genes in the regulation networks of miRNA-mRNA.

\section{Supplementary Files}

This is a list of supplementary files associated with this preprint. Click to download.

- GraphicalAbstract.png 